\title{
Gauge invariance in teleparallel gravity theories: A solution to the background structure problem
}

\author{
E. Minguzzi \\ Dipartimento di Fisica dell'Università di Milano-Bicocca, \\ Piazza della Scienza 3, 20126 Milano, Italy
}

\begin{abstract}
We deal with the problem of identifying a background structure and its perturbation in tetrad theories of gravity. Starting from a peculiar trivial principal bundle we define a metric which depends only on the gauge connection. We find the allowed four-dimensional structure groups; two of them turn out to be the translation group $T_{4}$ and the unitary group $U(2)$. When the curvature vanishes the metric reduces to its background form which coincides with Minkowski flat metric for the $T_{4}$ case and with the Einstein static universe metric for the $U(2)$ case. The perturbation has a coordinate independent definition and allows for the introduction of observables distinguished from those obtained from the metric alone. Finally, we show that any teleparallel theory of gravity, and hence general relativity, can be considered as a gauge theory over the groups introduced.
\end{abstract}

PACS numbers: $04.20 . \mathrm{Cv}, 04.50 .+\mathrm{h}$

\section{INTRODUCTION}

Teleparallel theories of gravity have a venerable history. In 1928 Einstein [1] introduced the notion of absolute parallelism in his attempt of unifying gravity and electromagnetism. That project failed but later [2, 3] the idea of a teleparallel geometry was revived as a geometrical alternative to the Riemmanian approach of general relativity. Those investigations led to valuable results particularly in connection with the study of energymomentum, its covariance, positivity and localization [4, []. In 1967 Hayashi and Nakano [6] showed that teleparallel gravity can be seen as a gauge theory over the translation group [7, 8 with a Lagrangian quadratic in the strength tensor. Some author started to consider modifications of the teleparallel equivalent of general relativity thus discovering a one parameter family of quadratic Lagrangians which reproduce the correct low energy behavior [9]. Such teleparallel theories are experimentally indistinguishable from Einstein's gravity even if, from the theoretical side, they are not invariant under local Lorentz trasformations of the tetrad field. Moreover, the one parameter family cannot be altered with a slight modification of the coefficients without introducing ghosts 10], and the parameter, for the same reason, should be taken to be positive. Thus, Kopczyński's 11] proof that such Lagrangians were unable to determine uniquely the evolution of the teleparallel geometry was taken as a serious drawback even if, as shown later by Nester [12], it was less severe than expected and limited to special solutions. Who takes this problem seriously has to drop the teleparallel geometry and has to consider it as auxiliary: an interpretation useful to write down Lagrangians but not realized in nature. In this case the matter Lagrangian should be invariant under the hidden symmetries of the gravitational Lagrangian. In particular the coupling of spinor matter fields with gravity should be written through the Levi-Civita connection, not Weitzenböck's [11, 13, 14. This solution is not completely satisfying because even if we can introduce a stress-energy tensor of gravity which is covariant under coordinate transformations, global Lorentz transformations and gauge transformations [4], we cannot establish, for instance, the value of the energy density as seen in a local Lorentz frame. This is because the stress-energy tensors so far proposed are not invariant under local Lorentz transformations of the OT frame, and moreover the dynamics is not able to define a privileged OT frame because of the local hidden Lorentz symmetry of the family of viable Lagrangians 11, 14. Fortunately, this problem arises only in those special solutions studied by Nester. Alternatively, one can remove altogether the lack of determinism adding to the Lagrangian higher order terms in the torsion [10. This, however, not only means a more radical departure from general relativity (at least from the theoretical side), but also from the Yang-Mills theory that the gauge interpretation of teleparallel theory so strongly resembles.

Here we study the teleparallel theories as Yang-Mills theories even if with a dependence of the Lagrangian on the curvature somewhat more complicated. Our task is to show that the gauge formulation of teleparallel gravity theories allows a solution of the background structure problem. Ultimately this can be considered as the true reason for the success of these theories in dealing with the stress-energy tensor. We shall deal only with the one parameter family of experimentally viable teleparallel theories, relying on the fact that an unpredictable behavior, if present, can be removed with a slight perturbation of the initial value data [12]. One can also get rid of it by assuming that a higher order term in the curvature was implicitly added to the Lagrangian.

We use the Greek alphabet $(\mu, \nu, \rho, \ldots=0,1,2,3)$ to denote indices related to spacetime, and the Latin alphabet $(\mathrm{a}, \mathrm{b}, \mathrm{c}, \ldots=0,1,2,3)$ to denote indices related to the internal space. The flat spacetime metric $\eta_{a b}$ is fixed by $\eta_{00}=1$ and the totally antisymmetric tensor is normalized by $\epsilon_{0123}=1$. We use the natural units: $\hbar=1$, $c=1$. 


\section{BACKGROUND IDENTIFICATION}

The identification of a background structure in theories invariant under diffeomorphisms is notoriously difficult. This is an important issue because in turn, in quantum field theory, the excitations with respect to a fixed background receive a particle interpretation [15]. Hence the problem of identifying a background structure is central in quantum gravity. One can also avoid this problem taking unaltered the spirit of general relativity. A program of quantization of this kind can be performed and leads to canonical gravity [16]. Before we abandon the idea of a privileged background structure let us look more closely to the problem of its definition.

In metric theories of gravity one can try to introduce by hand a background structure rewriting the metric in the form

$$
g_{\mu \nu}=\eta_{\mu \nu}+h_{\mu \nu},
$$

and identifying $\eta_{\mu \nu}$ with the background and $h_{\mu \nu}$ with the perturbation. This can be justified if we are studying a spacetime which is Minkowskian at spatial infinity but is still not satisfying. The splitting of the metric in background and perturbation parts turns out to be dependent on the coordinate system chosen. Let two observers label events in different ways with systems of coordinates $\left\{x^{\mu}\right\}$ and $\left\{x^{\prime \mu}\right\}$ respectively. Even if the two systems of coordinates coincide at spatial infinity, the associated backgrounds $\eta_{\mu \nu} \mathrm{d} x^{\mu} \mathrm{d} x^{\nu}$ and $\eta_{\mu \nu} \mathrm{d} x^{\prime \mu} \mathrm{d} x^{\prime \nu}$ differ. The prescription (11) introduces a background in a non-covariant way with respect to coordinate changes. It cannot be used in many circumstances: for instance in quantum gravity where we cannot arbitrarily privilege a system of coordinates [19]. We can reach the same conclusion even if we look for scalars that can be constructed from $\eta$ and $h$. It is not difficult to show that the only scalars that can be constructed this way are exactly those that can be constructed using the full metric $g_{\mu \nu}$ alone. This means, because any observable should be coordinate independent, that the splitting proposed is artificial. Any observable expresses properties of the full geometry, nothing can be said about the background metric and its perturbation separately. A better definition would be

$$
\begin{aligned}
g_{\mu \nu} & =\bar{g}_{\mu \nu}+h_{\mu \nu}, \\
\bar{R}_{\beta \mu \nu}^{\alpha} & =0 .
\end{aligned}
$$

It says that a coordinate system where (1) holds exists without, however, fixing it from the beginning. The dynamics should determine that coordinate system starting from the initial value data. Unfortunately, this prescription is not well suited for practical calculations.

Let us consider a principal fiber bundle $P$ with structure group $G$ and let the spacetime $M$ be the base. Over $P$ let us consider a flat connection $\tilde{\omega}$ and a second connection $\omega$. Then, clearly, if $M$ is simply connected, the fiber bundle is trivial. A structure like this is familiar in teleparallel theories where $P$ is the bundle of linear frames and the two connections are given by Weitzenböck's and Levi-Civita's respectively. Here, however, this structure acquires a different role. We require $G$ to be a 4 -dimensional Lie group and we define the metric of $M$ with

$$
g_{\mu \nu} \mathrm{d} x^{\mu} \mathrm{d} x^{\nu}=a^{2} I(\tilde{A}-A, \tilde{A}-A) .
$$

$I$ is a symmetric, bilinear and ad-invariant function defined on the Lie algebra of the structure group, $I: \mathcal{G} \times \mathcal{G} \rightarrow$ $\mathbb{R}$, and $\tilde{A}_{\mu}, A_{\mu}$, are the potentials of the connections in a given section $\sigma(x)$, e.g.: $A=\tau_{a} A_{\mu}^{a} \mathrm{~d} x^{\mu}=\sigma^{*} \omega$. The constant $a$ has the dimension of a length; its presence gives to $A_{\mu}^{a}$ the dimension of a mass. Under gauge transformations the difference of two potential transforms with the adjoint representation. Since $I$ is ad-invariant the metric so defined is gauge invariant.

The function $I$ is a metric for the Lie algebra $\mathcal{G}$. It must be Minkowkian because $g_{\mu \nu}$ is Minkowskian too. We can chose a base of generators $\tau_{a}$ such that

$$
I\left(\tau_{a}, \tau_{b}\right)=\eta_{a b}
$$

The requirement of ad-invariance for $I$ leads us to the classification of the allowed four-dimensional groups

$$
I\left(\left[\tau_{a}, \tau_{b}\right], \tau_{c}\right)+I\left(\tau_{b},\left[\tau_{a}, \tau_{c}\right]\right)=0 \Rightarrow f_{a b c}=f_{[a b c]},
$$

where $\left[\tau_{a}, \tau_{b}\right]=f_{a b}^{c} \tau_{c}$ and $f_{a b c}=\eta_{a d} f_{b c}^{d}$. Let us rewrite $f_{a b c}=v^{e} \epsilon_{e a b c}$. With a Lorentz transformation of the generators we can recast $v^{a}$ in a canonical form [17, moreover the residual parameter can be eliminated with a suitable rescaling of $\tau_{a}, I, a^{2}$, in such a way that the product $a^{2} I$ and Eq. (5) are left unchanged. Finally, the Lie algebras involved are

a) $v^{a}=0:$ Lie algebra of the translation group $T_{4}$

$$
f_{a b}^{c}=0 .
$$

b) $v^{2}>0$ : Lie algebra of the group $U(2)$

$$
f_{a b}^{c}=2 \epsilon_{0 a b d} \eta^{d c} .
$$

c) $v^{2}<0$ : Lie algebra of the group $G L(2, \mathbb{R})$

$$
f_{a b}^{c}=2 \epsilon_{3 a b d} \eta^{d c} .
$$

d) $v^{2}=0$ : Lie algebra of the two-dimensional Euclidean group with central charge

$$
f_{a b}^{c}=\epsilon_{0 a b d} \eta^{d c}+\epsilon_{3 a b d} \eta^{d c} .
$$

Notice that the generator $v^{a} \tau_{a}$ belongs to the center of the group. In the last case it plays the role of central charge for the Lie algebra of the Euclidean group in two dimensions $E_{2}$. 
From Eq. (5) it follows that the adjoint representation acts as a subgroup $H$ of the Lorentz group. Moreover if $v^{a} \neq 0, H$ is the little group of the four-vector $v^{a}$, since the structure coefficients are left unchanged after an adjoint transformation. For $G=T_{4}$ this subgroup is the trivial group $H=e$, for $G=U(2)$ it is $S O(3)$, for $G=G L(2, \mathbb{R})$ it is $S O(2,1)$ and in the last case it is $E_{2}$.

Let $\tilde{\sigma}(x)$ be an horizontal section with respect to the flat connection $\tilde{\omega}$ and let $\phi(x)$ be the transition function between the two sections $\sigma(x) \phi^{-1}(x)=\tilde{\sigma}(x)$ then $\tilde{A}=$ $\sigma^{*} \tilde{\omega}=\phi^{-1}(x) \mathrm{d} \phi(x)$. Finally,

$$
g_{\mu \nu} \mathrm{d} x^{\mu} \mathrm{d} x^{\nu}=a^{2} I\left(\phi^{-1} \mathrm{~d} \phi(x)-A, \phi^{-1} \mathrm{~d} \phi(x)-A\right)
$$

and the tetrad field can be defined to be

$$
\tau_{a} e_{\mu}^{a} \mathrm{~d} x^{\mu}=a\left(\tau_{a} A_{\mu}^{a} \mathrm{~d} x^{\mu}-\phi^{-1} \mathrm{~d} \phi(x)\right) .
$$

Notice that $\phi(x)$ is determined only up to global left multiplications $\phi(x) \rightarrow u \phi(x)$ because of the arbitrariness of $\tilde{\sigma}(x)$. This does not effect (12) which depends only on the combination $\phi^{-1} \mathrm{~d} \phi$.

The degrees of freedom given by the field $\phi$ are easily removed with a gauge transformation that sends $\phi$ to the identity. Let us call such gauge "OT gauge" because of its connection, that we shall exploit, with the OT frame. In the OT gauge the tetrad field and the potential $A_{\mu}^{a}$ are proportional. The potential in the OT gauge is our dynamical variable; in what follows we shall assume that the dynamics determines this field completely. Clearly, this is not the case if the Lagrangian is constructed from the metric alone, indeed the tetrad field is determined only up to a local Lorentz transformation. Like in teleparallel theories, we have to consider modifications to the general relativistic Lagrangian.

Let us return to the main question to show that (11) splits the metric in background and perturbation parts in a coordinate and gauge independent way. We identify the perturbation with the potential $A$ and the background metric with

$$
g_{B}=a^{2} I(\theta, \theta)
$$

where $\theta$ is the canonical 1 -form of the group $G \sqrt{18} \mid$. When the perturbation is a pure gauge $A=U^{-1} \mathrm{~d} U(x)$ the potential can be sent to zero through a gauge transformation. Then the metric reduces to $\phi(x)^{*} g_{B}$ that is, apart from a pullback which amounts simply to a coordinate transformation [20], the metric takes its background form. In other words, if $F$ is the curvature tensor,

$$
F=0 \Rightarrow g=\phi^{*} g_{B}
$$

The coordinate system in which $g=g_{B}$ is not fixed by the requirement $F=0$, indeed it depends on the map $\phi(x)$ that in turn depends on the value of $U(x)$. This shows that the equation $F=0$ is a coordinate independent way to state the equivalence of the metric with its background form. Here the splitting is not artificial because we can construct quantities like $F_{\mu \nu}^{a} F_{\alpha \beta}^{b} \eta_{a b} g^{\mu \alpha} g^{\nu \beta}$ that are scalars and gauge invariant. Moreover they cannot be recovered from the metric alone. Their gauge invariance is assured because the tetrad field (12) and the curvature transform, under gauge transformations, with the adjoint representation which, as we have seen, is a subgroup of the Lorentz group.

Before we start studying the dynamics let us esplicitate the background metric for the structure groups $T_{4}$ and $U(2)$.

a) $T_{4}$ : With the parameterization $\phi=\mathrm{e}^{-\tau_{a} \phi^{a}}$ the canonical 1-form becomes $\theta=\phi^{-1} \mathrm{~d} \phi=-\tau_{a} \mathrm{~d} \phi^{a}$ and the background metric

$$
g_{B}=a^{2} \eta_{a b} \mathrm{~d} \phi^{a} \mathrm{~d} \phi^{b}
$$

coincides with the Minkowski metric.

b) $U(2)$ : With the parameterization

$$
\phi=\mathrm{e}^{\lambda \tau_{0}} \mathrm{e}^{\chi\left(\tau_{1} \sin \theta \cos \varphi+\tau_{2} \sin \theta \sin \varphi+\tau_{3} \cos \theta\right)},
$$

and the representation (the metric is independent from the representation of the Lie algebra chosen) $\tau_{\mu}=i \sigma_{\mu}, \sigma_{0}=\mathrm{I}$, we find $I(\alpha, \alpha)=-\operatorname{det}(\alpha), \alpha \in \mathcal{G}$ and

$$
g_{B}=a^{2}\left\{\mathrm{~d} \lambda^{2}-\mathrm{d} \chi^{2}-\sin ^{2} \chi\left(\mathrm{d} \theta^{2}+\sin ^{2} \theta \mathrm{d} \varphi^{2}\right)\right\} .
$$

Hence, for $G=U(2)$ the background metric coincides with that of Einstein's static universe.

Analogous calculations lead to the background metric for the cases c) and d). They, however, do not enjoy the cosmological principle.

To summarize, we have identified the perturbation with the potential of a gauge theory. Its transformation under gauge and coordinates change are well known. From that variable one can construct scalars that express properties of the perturbation with respect to a background structure. The scalars to be considered depend on the supposed background structure, for instance, they are invariant under $U(2)$ gauge transformations in an Einstein's static universe background.

\section{DYNAMICS AND TELEPARALLEL THEORIES}

Now we have to construct a dynamics for the gauge potential. Let $\hat{A}_{\mu}^{a}$ be the potential in the OT gauge and let $\hat{e}_{\mu}^{a}$ be the tetrad field (12) in the same gauge: $\hat{e}_{\mu}^{a}=$ $a \hat{A}_{\mu}^{a}$. We introduce a teleparallel geometry on $M$ of OT frame $\left\{\hat{e}_{\mu}^{a}\right\}$, where the brackets recall that the OT frame is defined up to a global Lorentz transformation. We can rewrite the usual expression of the torsion in terms of the OT frame

$$
T_{\mu \nu}^{\rho}=\hat{e}_{a}^{\rho}\left(\partial_{\mu} \hat{e}_{\nu}^{a}-\partial_{\nu} \hat{e}_{\mu}^{a}\right)
$$


in a gauge invariant way

$$
T_{\mu \nu}^{\rho}=a e_{c}^{\rho} F_{\mu \nu}^{c}-\frac{1}{a} e_{c}^{\rho} f_{a b}^{c} e_{\mu}^{a} e_{\nu}^{b},
$$

where the tetrad field is given by Eq. (12) and where

$$
F_{\mu \nu}^{a}=\partial_{\mu} A_{\nu}^{a}-\partial_{\nu} A_{\mu}^{a}+f_{b c}^{a} A_{\mu}^{b} A_{\nu}^{c} .
$$

In order to shorten the notation let $F_{a b c}=\eta_{a d} F_{\mu \nu}^{d} e_{b}^{\mu} e_{c}^{\nu}$ and $T_{a b c}=e_{c \rho} T_{\mu \nu}^{\rho} e_{b}^{\mu} e_{c}^{\nu}$. Eq. (16) becomes

$$
T_{a b c}=a F_{a b c}-\frac{1}{a} f_{a b c},
$$

and the one parameter family of viable teleparallel Lagrangians [9, 10]

$$
\begin{aligned}
\mathcal{L} & =\frac{\sqrt{-g}}{16 \pi G}\left\{\frac{1}{4} T_{\alpha \mu \nu} T^{\alpha \mu \nu}+\frac{1}{2} T_{\alpha \mu \nu} T^{\mu \alpha \nu}+\right. \\
& \left.-T^{\mu \alpha \alpha} T_{\nu}^{\nu}{ }^{\alpha}+\alpha \frac{3}{2} T_{[\alpha \mu \nu]} T^{[\alpha \mu \nu]}\right\}
\end{aligned}
$$

becomes

$$
\begin{aligned}
& \mathcal{L}=\frac{\sqrt{-g}}{16 \pi \sigma^{2}}\left\{\frac{1+2 \alpha}{4} F_{a b c} F^{a b c}+\frac{1-2 \alpha}{2} F_{a b c} F^{b a c}+\right. \\
& \left.-F_{a b}^{a} F_{c}^{c b}+\frac{1-6 \alpha}{2} F_{a b c} f^{a b c}-\frac{1-6 \alpha}{4 a^{2}} f_{a b c} f^{a b c}\right\} .
\end{aligned}
$$

The linearized theory is ghost-free when the parameter $\alpha$ is positive [9]: $\alpha \geq 0$. General relativity corresponds to $\alpha=0$. The first term in Eq. (20) is the well known YangMills Lagrangian; its dimensionless coupling constant is given by

$$
\sigma=\frac{L_{P}}{a} .
$$

The reader should keep in mind that this similarity is only apparent: here even the metric and the tetrad field depend on the potential A; this leaves us with a theory that is still not renormalizable. The role of Eq. (20) is to exhibit the gauge invariance of teleparallel gravity theories, and hence of general relativity, under any of the four dimensional group studied in the previous section. Actually, we have obtained this gauge invariance with the introduction of a new degree of freedom given by the field $\phi(x)$. One can correctly suspect that the need for the introduction of a new field is a signal that the symmetries developed are not genuine new symmetries of the original Lagrangian. Moreover, one can be concerned about the fact that our dynamical variable is the potential in the OT gauge: if in practical calculation we have to return to the usual tetrad formulation, what is the advantage of introducing such dependence of the metric? To answer these questions we need to introduce the internal coordinate representation.

So far, we have used the spacetime coordinates. Let us choose a gauge such that $\phi: M \rightarrow G$ is injective. In such a gauge we can perform a coordinate transformation from the spacetime coordinates $\left\{x^{\mu}\right\}$ to given internal coordinates $\left\{\phi^{a}\right\}$ of the group manifold $G$. For example the Lagrangian in the internal coordinates becomes $\phi^{-1 *} \mathcal{L}(x)$ with a tetrad field given by 21]

$$
\tau_{a} e_{b}^{a}\left(\phi^{a}\right) \mathrm{d} \phi^{a}=\tau_{a} A_{b}^{a}\left(\phi^{a}\right) \mathrm{d} \phi^{b}-\theta .
$$

From this equation we see that the field $\phi(x)$ has been completely removed by the coordinate change. In the internal coordinate representation the gauge symmetry turns out to be an alternative way of recasting the invariance under coordinate transformations. To any coordinate change in the internal formalism corresponds a gauge transformation in the spacetime formalism; the converse, however, is not true. For instance the OT gauge can not be accomplished in the internal formalism because the field $\phi(x)$, in that case, is not injective. In the internal representation the invariance under coordinate transformation and the invariance under gauge transformation are linked: the transformation law for the potential becomes $\left(\sigma^{\prime}=\sigma u\right)$

$$
\tau_{a}{A^{\prime}}_{c}^{a}=\left\{u^{-1} \tau_{a} A_{b}^{a} u+u^{-1} \partial_{b} u\right\} \frac{\partial \phi^{b}}{\partial{\phi^{\prime}}^{c}},
$$

and the transformation law for the curvature becomes

$$
F_{a b}^{\prime}=u^{-1} F_{c d} u \frac{\partial \phi^{c}}{\partial{\phi^{\prime}}^{a}} \frac{\partial \phi^{d}}{\partial{\phi^{\prime}}^{b}},
$$

where the matrix $u(\phi)$ is related to the transformation $\phi^{\prime a}\left(\phi^{b}\right)$ by the product $\phi^{\prime}=\phi u(\phi)$. In the same way it can be shown, for example, that the metric given by (11) transform as a tensor under (23). Notice that in the internal formalism there is no distinction between internal and spacetime indices because in the spacetime manifold we have introduced internal coordinates.

Let us investigate more closely the case $G=T_{4}$. In the previous section we defined the coordinates $\left\{\phi^{a}\right\}$ on the group manifold: $\phi=\mathrm{e}^{-\tau_{a} \phi^{a}}$. The tetrad field in the spacetime formalism is [6]

$$
e_{\mu}^{a}=\partial_{\mu} \phi^{a}(x)+A_{\mu}^{a}(x),
$$

whereas in the internal formalism is (Eq. (22))

$$
e_{b}^{a}(\phi)=\delta_{b}^{a}+A_{b}^{a}(\phi) .
$$

Apart for changes due to the notation, this is exactly the dependence of the tetrad field on the potential given in [7]. Indeed, our formalism in the $G=T_{4}$ case gives rise to the widely studied translational gauge symmetry of teleparallel theories. In literature it has been studied both in internal coordinates [7] and in spacetime coordinates [4, 6].

Now we see the advantage of these gauge formulations. In the internal coordinate representation, contrary to what happens in the spacetime representation, the Lagrangian does not reduce to its usual tetrad form because we are not working (and we cannot work) in the 
OT gauge. Moreover, the dynamical variable is given by the potential $A_{b}^{a}(\phi)$ and there is no further degree of freedom apart from that of making gauge-coordinate transformations. The metric depends on the potential through the tetrad field (22). The identification of the potential $A(\phi)$ with the perturbation is coordinate independent, its transformation law being Eq. (23).

As a final point let us investigate the invariance under global Lorentz transformations. We should expect that the formulation in terms of a perturbation spoils this explicit invariance see (Eq. (19)) if the background structure does not share the same symmetry. This is indeed the case. Only the structure coefficients of the group $G=T_{4}$ are left unchanged under the replacement $\tau_{a}=\Lambda_{a}^{b^{\prime}} \tau_{b^{\prime}}$. For this group one can accomplish the global Lorentz invariance with $\phi^{a}=\Lambda_{b^{\prime}}^{a} \phi^{b^{\prime}}$ and $A_{\mu}^{a}(x)=\Lambda_{b^{\prime}}^{a} A_{\mu}^{b^{\prime}}(x)$. Of course, in the internal representation even the coordinates transform and we have

$$
A_{b}^{a}(\phi)=\Lambda_{c^{\prime}}^{a} A_{d^{\prime}}^{c^{\prime}}(\phi) \Lambda_{b}^{d^{\prime}} .
$$

Surprisingly, in the previous literature, despite of the properties of the gauge formulation, most of the calculations were performed in the usual tetrad formalism. This, however, is quite natural if one looks for non-perturbative results.

\section{CONCLUSIONS}

In order to solve the background structure problem of gravity theories, we proposed a metric of the form (11) where two connections were introduced. We showed that, if the dynamics determines the potential of the gauge the- ory completely (up to gauge or coordinate changes), the splitting of the metric in background and perturbation parts is physical since new observables, not dependent from the full metric alone, can be constructed. We classified the allowed four-dimensional structure groups finding suitable backgrounds for the $T_{4}$ and $U(2)$ cases. We went to exploit the dynamics with the introduction of a teleparallel geometry on the manifold. Taking advantage of teleparallel gravity results, that geometry was used to construct Lagrangians compatible with the low energy limit of general relativity. It should be mentioned, however, that the gauge approach allows a more large variety of gravitational Lagrangians than the teleparallel approach. For instance the Lagrangian $F_{\mu \nu}^{a} F_{\alpha \beta}^{b} \delta_{a b} g^{\mu \alpha} g^{\nu \beta}$ is coordinate and gauge independent but has no teleparallel analogue. Teleparallel Lagrangians have the advantage of being invariant under global Lorentz transformation. This still seems a necessary condition for the introduction of spinor matter fields. Finally, teleparallel theories were interpreted as gauge theories over different backgrounds in dependence of the structure group chosen. Most interesting cases were the Minkowski flat metric and the Einstein static universe. As a final comment, Eq. (21) suggests that renormalization can effect the 'radius' of the universe. Of course, the theory is not renormalizable and this is only a speculation, anyway it seems to deserve further investigations.

\section{Acknowledgments}

I wish to thank C. Destri for useful discussions and for a critical reading of the manuscript, and INFN for financial support.
[1] A. Einstein, Sitzungsber. Preuss. Akad. Wiss. Phys.Math. Klasse 224 (1928).

[2] C. Möller, K. Dan. Vidensk. Selsk. Math. Fys. Skr. 1 (1961).

[3] C. Pellegrini and J. Plebanski, K. Dan. Vidensk. Selsk. Mat. Fys. Skr. 2 (1962).

[4] V. C. de Andrade, L. C. T. Guillen, and J. G. Pereira, Phys. Rev. Lett. 84, 4533 (2000).

[5] J. M. Nester, Int. J. Mod. Phys. A 4, 1755 (1989).

[6] K. Hayashi and T. Nakano, Prog. Theor. Phys. 38, 491 (1967).

[7] Y. M. Cho, Phys. Rev. D 14, 2521 (1976).

[8] V. C. de Andrade and J. G. Pereira, Phys. Rev. D 56, 4689 (1997).

[9] K. Hayashi and T. Shirafuji, Phys. Rev. D 19, 3524 (1979).

[10] F. Müller-Hoissen and J. Nitsch, Gen. Relativ. Gravit. 17, 747 (1985).

[11] W. Kopczyński, J. Phys. A 15, 493 (1982).

[12] J. M. Nester, Class. Quantum Grav. 5, 1003 (1988).

[13] V. C. de Andrade, L. C. T. Guillen, and J. G. Pereira, Phys. Rev. D 64, 027502 (2001).
[14] K. Hayashi and T. Shirafuji, Phys. Rev. D 24, 3312 (1981).

[15] J. W. Maluf, J. Math. Phys. 35, 335 (1994).

[16] A. Ashtekar, Lectures on Non-Perturbative Canonical Gravity, vol. 6 of Advanced Series in Astrophysics and Cosmology (World Scientific, Singapore, 1991).

[17] S. Weinberg, The Quantum Theory of Fields, vol. 1 (Cambridge University Press, Cambridge, 1995).

[18] S. Kobayashi and K. Nomizu, Foundations of Differential Geometry, vol. I of Interscience tracts in pure and applied mathematics (Interscience Publishers, New York, 1963).

[19] To privilege a coordinate system means to privilege a way of labeling events. For instance, an observer can take minus the redshift as time variable for the light emission from distant stars, whereas another observer can take, as time variable, the distance obtained with the distanceredshift relation.

[20] The coordinate transformation can be singular. In that case the metric turns out to be degenerate. This does not happen if the condition $F \approx 0$ in some region of spacetime follows from the dynamics and if the dynamics itself guarantees the metric to be not degenerate. 
[21] For clearness, in internal coordinates the reciprocal of $e_{b}^{a}$ should be written $e^{-1 b}$. 